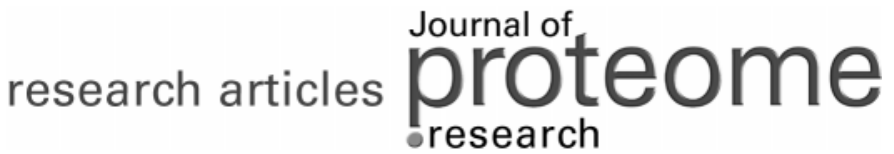

\section{Proteomic Analysis of Peritoneal Dialysate Fluid in Patients with Different Types of Peritoneal Membranes}

\author{
Suchai Sritippayawan, ${ }^{\dagger}$ Wararat Chiangjong, ${ }^{\ddagger}$ Theptida Semangoen,,$\stackrel{\$}{ }$ Nipa Aiyasanon, ${ }^{\dagger}$ \\ Parnthip Jaetanawanitch, ${ }^{\dagger}$ Supachok Sinchaikul, ${ }^{\perp}$ Shui-Tein Chen, ${ }^{\perp, \#}$ \\ Somkiat Vasuvattakul, ${ }^{\dagger}$ and Visith Thongboonkerd ${ }^{*, \dagger}$
}

\begin{abstract}
Division of Nephrology, Department of Internal Medicine, Medical Molecular Biology Unit, Office for Research and Development, and Department of Immunology and Immunology Graduate Program, Faculty of Medicine, Siriraj Hospital, Mahidol University, Bangkok, Thailand, Institute of Biological Chemistry and Genomic Research Center, Academia Sinica, Taipei, Taiwan, and Institute of Biochemical Sciences, College of Life Science, National Taiwan University, Taipei, Taiwan
\end{abstract}

Received May 20, 2007

\begin{abstract}
Efficacy of peritoneal dialysis is determined by solute transport through peritoneal membranes. With the use of the peritoneal equilibration test (PET), peritoneal membranes can be classified as high $(\mathrm{H})$, high average (HA), low average (LA), and low (L) transporters, based on the removal or transport rate of solutes, which are small molecules. Whether there is any difference in macromolecules (i.e., proteins) removed by different types of peritoneal membranes remains unclear. We performed a gel-based differential proteomics study of peritoneal dialysate effluents (PDE) obtained from chronic peritoneal dialysis (CPD) patients with $\mathrm{H}, \mathrm{HA}, \mathrm{LA}$, and $\mathrm{L}$ transport rates $(n=5$ for each group; total $n=20)$. Quantitative analysis and ANOVA with Tukey's posthoc multiple comparisons revealed five proteins whose abundance in PDE significantly differed among groups. These proteins were successfully identified by matrix-assisted laser desorption ionization quadrupole time-of-flight (MALDI-Q-TOF) mass spectrometry (MS) and tandem mass spectrometry (MS/MS) analyses, including serum albumin in a complex with myristic acid and triiodobenzoic acid, $\alpha 1$-antitrypsin, complement component C4A, immunoglobulin $\kappa$ light chain, and apolipoprotein A-I. The differences among groups in PDE levels of C4A and immunoglobulin $\kappa$ were clearly confirmed in a validation set of the other 24 patients $(n=6$ for each group) using ELISA. These data may lead to better understanding of the physiology of peritoneal membrane transport in CPD patients. Extending the study to a larger number of patients with subgroup analyses may yield additional information of the peritoneal dialysate proteins in association with dialysis adequacy, residual renal function, nutritional status, and risk of peritoneal infection.
\end{abstract}

Keywords: dialysate $\bullet$ kidney $\bullet$ membranes $\bullet$ peritoneal dialysis $\bullet$ proteome $\bullet$ renal failure $\bullet$ solute clearance

\section{Introduction}

Solute transport and ultrafiltration (UF) rates vary largely in patients with end-stage renal disease who are treated with chronic peritoneal dialysis (CPD). ${ }^{1}$ This information reflects the differential capability of peritoneal membranes to transport or remove solutes and water in individual patients. With the use of the standard peritoneal equilibration test (PET), peritoneal membranes can be classified into four main types on the basis of the transport rates, including high $(\mathrm{H})$, high average (HA),

\footnotetext{
* To whom correspondence should be addressed. Phone/Fax: +66-2 4184793. E-mail: thongboonkerd@dr.com (or) vthongbo@yahoo.com.

${ }^{\dagger}$ Division of Nephrology, Department of Internal Medicine, Mahidol University.

${ }^{\ddagger}$ Medical Molecular Biology Unit, Office for Research and Development, Mahidol University.

$\S$ Department of Immunology and Immunology Graduate Program, Mahidol University.

${ }^{\perp}$ Academia Sinica.

" National Taiwan University.
}

4356 Journal of Proteome Research 2007, 6, 4356-4362

Published on Web 10/09/2007 low average (LA), and low (L) transporters. ${ }^{1}$ This classification is based primarily on the removal of solutes, which are small molecules. Several studies have shown an association of the type of peritoneal membranes and the outcome of CPD. ${ }^{2}$ The high transporters are associated with greater loss of UF and higher risk of mortality. ${ }^{3}$ Several factors may be involved in this different outcome, including UF rate, solute clearance, inflammation, and nutritional status. Recently, there is evidence demonstrating that patients with high transport membranes have greater peritoneal protein loss. ${ }^{4}$ However, whether there is any difference in the component of proteins removed by different types of peritoneal membranes remains unclear.

This study was therefore conducted to examine proteome profiles and identify differentially expressed proteins in peritoneal dialysate effluents (PDE) of $20 \mathrm{CPD}$ patients with different types of peritoneal membranes. Gel-based, differential proteomics approach revealed five protein spots whose intensity levels significantly differed among groups. These protein 
spots were then successfully identified by matrix-assisted laser desorption ionization quadrupole time-of-flight (MALDI-QTOF) mass spectrometry (MS) and tandem mass spectrometry (MS/MS) analyses. Their potential roles in the physiology of peritoneal membrane transport in CPD patients are discussed. The differences among groups in PDE levels of C4A and immunoglobulin $\kappa$ light chain were clearly confirmed in a validation set of the other 24 patients using ELISA.

\section{Materials and Methods}

Patients. A total of 44 CPD patients, who had different types of peritoneal membranes, were recruited into this study. The dialysate fluid containing $\mathrm{Na}^{+}$(132 mequiv/L), $\mathrm{Ca}^{2+}(3.5$ mequiv/L), $\mathrm{Mg}^{2+}\left(0.5\right.$ mequiv/L), $\mathrm{Cl}^{-}$(96 mequiv/L), lactate $(40$ mequiv/L), and dextrose (1.5\%) with the osmolarity of 346 mOsmol/L (Dianeal PD-2; Baxter; Deerfield, IL) was used throughout the study. All subjects were free from peritonitis at least 1 month prior to the study, which was carried out in accordance with the Declaration of Helsinki (2000) of the World Medical Association and has been approved by the Institutional Ethics Committee. All these patients were grouped as H, HA, $\mathrm{LA}$, and $\mathrm{L}$ transporters, which were defined by dialysate/serum creatinine $(\mathrm{Cr})$ ratios during $4 \mathrm{~h}$ dwell time $\left(D / P_{\mathrm{Cr} 4 \mathrm{~h}}\right)$ according to the standard PET, as described by Twardowski. ${ }^{1}$

The test set containing 20 patients ( $n=5$ for each group) was subjected to proteomic analysis using a two-dimensional gel electrophoresis (2-DE) approach. Their clinical data, age, gender, duration of CPD therapy, and numbers of peritonitis episodes were reviewed and recorded. Peritoneal and serum urea nitrogen, Cr, and albumin were analyzed by an Express Plus autoanalyzer (CIBA Corning Diagnostics Corp., Norwood, MA). Total protein levels in PDE were analyzed by the biuret method. Peritoneal small solute clearances were determined by weekly peritoneal urea clearance normalized with distribution volume of urea ( $\mathrm{pKt} / V_{\text {urea }}$ ) and creatinine clearance normalized with body surface area (npCCr), as previously described. ${ }^{5}$ The distribution volume of urea $\left(V_{\text {urea }}\right)$ and body surface area were calculated using Watson and Hume equation and Dubois and Dubois formula, respectively. ${ }^{5}$ Nutritional status was assessed by patients' serum albumin and normalized protein nitrogen appearance (nPNA) as recommended in the European Best Practice Guidelines for peritoneal dialysis. ${ }^{6}$ Dialysate CA-125 level was also measured. After the PET and proteomic study, numbers of peritonitis episodes in individual patients were recorded for 1 year.

The validation set containing 24 patients ( $n=6$ for each group) was subjected to confirmation of the proteomic data using ELISA to measure PDE levels of C4A and IgG.

Sample Collection. The PDE samples were collected at the end of the PET study. Immediately after the collection (within 30 min interval), cells and debris were removed by low-speed centrifugation (1000g for $10 \mathrm{~min}$ ) and clear supernatants were saved at $-70{ }^{\circ} \mathrm{C}$ until use.

Sample Preparation for Proteomic Analysis. Proteins in the supernatant of PDE were precipitated with $75 \%$ ethanol and solubilized with $7 \mathrm{M}$ urea, $2 \mathrm{M}$ thiourea, 4\% 3-[(3-cholamidopropyl)dimethylamino]-1-propanesulfonate (CHAPS), $2 \%$ ampholytes, $120 \mathrm{mM}$ dithiothreitol (DTT), and $40 \mathrm{mM}$ Tris. Protein concentrations in individual samples were measured using the Bradford's method. Because urea, thiourea, CHAPS, and other compositions in the sample/lysis buffer can interfere with the protein estimation, we generated the standard curve using bovine serum albumin (BSA) at concentrations of 0,2 ,
5,7 , and $10 \mu \mathrm{g} / \mu \mathrm{L}$ in the same sample/lysis buffer to ensure that the standards and the samples had the same background that might occur due to chemical interference.

Two-Dimensional Gel Electrophoresis (2-DE) and Staining. An equal amount $(200 \mu \mathrm{g})$ of total protein from each sample was resolved in each 2-D gel ( $n=5$ gels in each group; total $n$ $=20$ gels). Immobiline DryStrip (linear $\mathrm{pH}$ gradient of 3-10, 7 cm long; GE Healthcare, Uppsala, Sweden) was rehydrated overnight with $200 \mu \mathrm{g}$ of total protein that was premixed with a rehydration buffer containing $7 \mathrm{M}$ urea, $2 \mathrm{M}$ thiourea, $2 \%$ CHAPS, 2\% (v/v) ampholytes (pH 3-10), 120 mM DTT, 40 mM Tris-base, and bromophenol blue (to make the final volume of $150 \mu \mathrm{L}$ per strip). The first-dimensional separation (IEF) was performed in an Ettan IPGphor II IEF System (GE Healthcare) at $20{ }^{\circ} \mathrm{C}$, using stepwise mode to reach $9000 \mathrm{~V} \cdot \mathrm{h}$. After completion of the IEF, proteins on the strip were equilibrated in a buffer containing $6 \mathrm{M}$ urea, $130 \mathrm{mM}$ DTT, 30\% glycerol, $112 \mathrm{mM}$ Tris base, $4 \%$ sodium dodecyl sulfate (SDS), and $0.002 \%$ bromophenol blue for $10 \mathrm{~min}$ and then with another buffer containing $6 \mathrm{M}$ urea, $135 \mathrm{mM}$ iodoacetamide, 30\% glycerol, $112 \mathrm{mM}$ Tris base, $4 \%$ SDS, and $0.002 \%$ bromophenol blue for $10 \mathrm{~min}$. The IPG strip was then transferred onto $12 \%$ acrylamide slab gel $(8 \mathrm{~cm} \times 9.5 \mathrm{~cm})$, and the seconddimensional separation was performed in an SE260 MiniVertical Electrophoresis Unit (GE Healthcare) with the current of $20 \mu \mathrm{A} /$ gel for $1.5 \mathrm{~h}$. Separated protein spots were then visualized with coomassie brilliant blue R-250 stain (Fluka Chemica AG, Buchs, Switzerland).

Matching and Analysis of Visualized Protein Spots. Image Master 2D Platinum (GE Healthcare) software was used for matching and analysis of protein spots in 2-D gels. Parameters used for spot detection were (i) minimal area $=10$ pixels, (ii) smooth factor $=2.0$, and (iii) saliency $=2.0$. A reference gel was created from an artificial gel combining all of the spots presenting in different gels into one image. The reference gel was then used for matching the corresponding protein spots between gels. Background subtraction was performed, and the intensity volume of each spot was normalized with total intensity volume (summation of the intensity volumes obtained from all spots within the same 2-D gel).

In-Gel Tryptic Digestion. The protein spots whose intensity levels significantly differed among groups were excised from 2 -D gels, washed twice with $200 \mu \mathrm{L}$ of $50 \%$ acetonitrile (ACN)/ $25 \mathrm{mM} \mathrm{NH}_{4} \mathrm{HCO}_{3}$ buffer (pH 8.0) at room temperature for 15 min, and then washed once with $200 \mu \mathrm{L}$ of $100 \%$ ACN. After washing, the solvent was removed, and the gel pieces were dried by a SpeedVac concentrator (Savant; Holbrook, NY) and rehydrated with $10 \mu \mathrm{L}$ of $1 \%$ (w/v) trypsin (Promega; Madison, WI) in $25 \mathrm{mM} \mathrm{NH}_{4} \mathrm{HCO}_{3}$. After rehydration, the gel pieces were crushed with siliconized blue stick and incubated at $37^{\circ} \mathrm{C}$ for at least $16 \mathrm{~h}$. Peptides were subsequently extracted twice with $50 \mu \mathrm{L}$ of $50 \%$ ACN/5\% trifluoroacetic acid (TFA); the extracted solutions were then combined and dried with the SpeedVac concentrator. The peptide pellets were resuspended with 10

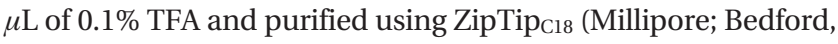
MA). The peptide solution was drawn up and down in the ZipTip $_{\mathrm{C} 18}$ for 10 times and then washed with $10 \mu \mathrm{L}$ of $0.1 \%$ formic acid by drawing up and expelling the washing solution for three times. The peptides were finally eluted with $5 \mu \mathrm{L}$ of $75 \%$ ACN $/ 0.1 \%$ formic acid.

Protein Identification by MALDI-Q-TOF MS and MS/MS Analyses. The proteolytic samples were premixed 1:1 with the matrix solution $(5 \mathrm{mg} / \mathrm{mL} \alpha$-cyano-4-hydroxycinnamic acid 
(CHCA) in 50\% ACN, 0.1\% v/v TFA, and 2\% w/v ammonium citrate) and spotted onto the 96-well sample stage. The samples were analyzed by the Q-TOF Ultima mass spectrometer (Micromass; Manchester, U.K.), which was fully automated with a predefined probe motion pattern and the peak intensity threshold for switching over from MS survey scanning to MS/ MS and from one MS/MS to another. Within each sample well, parent ions that met the predefined criteria (any peak within the $m / z$ 800-3000 range with intensity above 10 count \pm include/exclude list) were selected for CID MS/MS using argon as the collision gas and a mass dependent $\pm 5 \mathrm{~V}$ rolling collision energy until the end of the probe pattern was reached. The LM and HM resolution of the quadrupole were both set at 10 to give a precursor selection window of about 4 Da wide. Manual acquisition and optimization for individual samples or peaks was also possible.

The instrument was externally calibrated to $<5 \mathrm{ppm}$ accuracy over the mass range of $\mathrm{m} / z$ 800-3000 using a sodium iodide and PEG 200, 600, 1000, and 2000 mixtures and further adjusted with Glu-fibrinopeptide B as the near-point lock mass calibrant during data processing. At a laser firing rate of $10 \mathrm{~Hz}$, individual spectra from a $5 \mathrm{~s}$ integration period acquired for each of the MS survey and MS/MS performed were combined, smoothed, deisotoped (fast option), and centroided using the ProteinLynx GlobalSERVER 2.0 data processing software (Micromass). This entailed the identification of the monoisotopic, carbon-12 peaks for MS data, and deconvolution of multiply charged spectra to their singly charged equivalents for MS/ MS data. MaxEnt 3, a maximum-entropy-based technique, has been designed for this purpose and is an integral part of ProteinLynx GlobalSERVER 2.0.

The combined MS and MS/MS ion meta data were searched in concert against the NCBI human protein database using the ProteinLynx GlobalSERVER 2.0 workflow. The search algorithm employed a Hidden Markov model that incorporates empirically determined fragmentation characteristics to increase the efficacy of the search. Additionally, the MS and MS/MS data were extracted and outputted as the searchable .txt and .pkl files, respectively, for independent searches using the MASCOT search engine (http://www.matrixscience.com), assuming that peptides were monoisotopic, oxidized at methionine residues, and carbamidomethylated at cysteine residues. Only 1 missed trypsin cleavage was allowed, and peptide mass tolerances of 100 and $50 \mathrm{ppm}$ were used for peptide mass fingerprinting and MS/MS ions search, respectively. The search was performed using the NCBI human protein database. Significant hits required MS matching scores > 65 and/or MS/MS ion scores $>36$.

Measurements of PDE Levels of C4A and IgG using ELISA. The proteomic data were validated by ELISA to measure PDE levels of C4A and IgG in a validation set of samples $(n=6$ in each group; total $n=24)$. PDE samples $(10 \mathrm{~mL}$ from each patient) were lyophilized and resuspended in $500 \mu \mathrm{L}$ of $\mathrm{dI}$ water. The protein concentration was then measured using the Bradford's method. The protein concentrations of all samples were then adjusted to $50 \mu \mathrm{g} / \mathrm{mL}$ and $0.1 \mu \mathrm{g} / \mathrm{mL}$ for measurements of C4A and IgG, respectively.

To measure C4A level, $5 \mu \mathrm{g}$ of total protein derived from the PDE sample of each patient was coated overnight onto the 96well ELISA plate at $4{ }^{\circ} \mathrm{C}$. The sample was washed five times with a washing buffer containing $0.05 \%$ Tween-20 in PBS, and nonspecific bindings were blocked with $1 \%$ BSA in PBS at room temperature for $2 \mathrm{~h}$. The sample was washed five times and probed with goat antihuman C4A (sc-23483; Santa Cruz Biotechnology Inc.; Santa Cruz, CA) $(10 \mu \mathrm{g} / \mathrm{mL}$ in $0.1 \%$ BSA/PBS) at room temperature for $1 \mathrm{~h}$. After five washes, the sample was probed with rabbit antigoat IgG conjugated with horseradish peroxidase (P0449; DakoCytomation; Glostrup, Denmark) (1: 2000 in $0.1 \%$ BSA/PBS) for $1 \mathrm{~h}$ at room temperature in the dark. After the other five washes, color reaction was developed using $0.6 \mathrm{mg} / \mathrm{mL} \quad o$-phenylenediamine dihydrochloride substrate (Sigma-Aldrich; St. Louis, MO) and protein concentration was measured at $\lambda 492 \mathrm{~nm}$ using an ELISA microplate reader (Anthos HTII; Anthos Labtec Instruments GmbH; Salzburg, Austria).

To measure IgG level, rabbit antihuman IgG (A0423; DakoCytomation) $\left(0.25 \mu \mathrm{g} / \mathrm{mL}\right.$ in $0.015 \mathrm{M} \mathrm{Na}_{2} \mathrm{CO}_{3}$ with $0.03 \mathrm{M}$ $\mathrm{NaHCO}_{3}$ ) was coated overnight onto the 96-well ELISA plate at $4{ }^{\circ} \mathrm{C}$. After washing and blocking as described above, the PDE protein sample (10 ng of total protein; $0.1 \mu \mathrm{g} / \mathrm{mL}$ in $0.1 \%$ BSA/PBS) was added and incubated at room temperature for $1 \mathrm{~h}$. The sample was then washed five times and incubated further with rabbit antihuman IgG conjugated with horseradish peroxidase (P0214; DakoCytomation) $(0.25 \mu \mathrm{g} / \mathrm{mL}$ in $0.1 \% \mathrm{BSA} /$ PBS) for $1 \mathrm{~h}$ at room temperature in the dark. After the other five washes, color reaction and protein measurement were performed as described above.

Statistical Analysis. The mean values of individual parameters were compared among the four different transport groups by ANOVA with Tukey's posthoc multiple comparisons using the SPSS program (version 13.0). $P<0.05$ was considered statistically significant.

\section{Results}

All CPD patients were grouped as H, HA, LA, and L transporters, which were defined by dialysate/serum creatinine ratios during $4 \mathrm{~h}$ dwell time $\left(D / P_{\mathrm{Cr} 4 \mathrm{~h}}\right)$ according to the standard PET, as described by Twardowski. ${ }^{1}$ The test set containing 20 patients ( $n=5$ for each group) was subjected to proteomic analysis using the 2-DE approach. The demographic data of these 20 patients are shown in Table 1 . Their age, gender and duration of CPD therapy were comparable among the four groups (H, HA, LA, and L). At least three patients in each group had considerably significant residual renal function (urine output $>100 \mathrm{~mL} /$ day). Renal clearances of small solutes, as determined by $\mathrm{rKt} / V_{\text {urea }}$ and nGFR, were comparable among groups. No patient had UF failure (as defined by UF $<100 \mathrm{~mL}$ after $4 \mathrm{~h}$ dwell with $2.27 \%$ glucose during the standard PET study). The $\mathrm{H}$ and HA transporters had significantly greater protein loss in PDE, consistent with the data reported previously. ${ }^{4}$ As a result, their serum albumin levels tended to be lower than those of the LA and L transporters.

Weekly npCCr (normalized peritoneal creatinine clearance) tended to be highest in the $\mathrm{H}$ transporters. Although weekly $\mathrm{pKt} / V_{\text {urea }}, \mathrm{CA}-125$ (the marker of peritoneal mesothelial cell mass), nPNA (normalized protein nitrogen appearance), and number of peritonitis-free patients were not significantly different among groups, the $\mathrm{H}$ and $\mathrm{HA}$ transporters tended to have higher weekly pKt/ $V_{\text {urea }}$ and CA-125 levels. These data implicated that the $\mathrm{H}$ and $\mathrm{HA}$ transporters tended to have greater small solute clearance than the LA and L transporters because of the greater solute transport with adequate UF.

Malnourished patients (nPNA $<1 \mathrm{~g} / \mathrm{kg} /$ day) ${ }^{6}$ were more likely to have inadequate urea clearance (tKt $\left./ V_{\text {urea }}<1.7\right)$ (Chisquare $p=0.09$ ), whereas the patients who had total Kt/ $V_{\text {urea }}$ (tKt $\left./ V_{\text {urea }}\right) 1.7$ (adequate urea clearance) had significantly 
Table 1. Demographic Data of 20 CPD Patients in the 2-DE Proteomic Studya

\begin{tabular}{|c|c|c|c|c|c|}
\hline parameters & $\begin{array}{l}\text { high } \\
(\mathrm{H})\end{array}$ & $\begin{array}{l}\text { high average } \\
\text { (HA) }\end{array}$ & $\begin{array}{l}\text { low average } \\
\text { (LA) }\end{array}$ & $\begin{array}{l}\text { low } \\
\text { (L) }\end{array}$ & $p$ \\
\hline no. & 5 & 5 & 5 & 5 & NS \\
\hline age (years) & $58 \pm 18$ & $58 \pm 9$ & $58 \pm 15$ & $58 \pm 13$ & NS \\
\hline male/female & $3: 2$ & $3: 2$ & $3: 2$ & $3: 2$ & NS \\
\hline dialysis duration (days) & $278 \pm 183$ & $240 \pm 432$ & $197 \pm 183$ & $589 \pm 409$ & NS \\
\hline $24 \mathrm{~h}$ PDE protein $(\mathrm{g})$ & $9.2 \pm 4.6$ & $13.8 \pm 5.4$ & $6.8 \pm 1.6$ & $5.5 \pm 1.5$ & 0.014 \\
\hline serum albumin (g/dL) & $2.8 \pm 0.6$ & $3.0 \pm 0.3$ & $3.3 \pm 0.2$ & $3.4 \pm 0.3$ & 0.054 \\
\hline CA-125 (IU $/ \mathrm{mL})$ & $10.0 \pm 4.2$ & $11.5 \pm 4.0$ & $7.7 \pm 2.5$ & $8.5 \pm 2.0$ & NS \\
\hline weekly $\mathrm{pKt} / V_{\text {urea }}$ & $1.79 \pm 0.59$ & $1.81 \pm 0.31$ & $1.75 \pm 0.46$ & $1.48 \pm 0.59$ & NS \\
\hline weekly $\mathrm{rKt} / V_{\text {urea }}$ & $0.5 \pm 0.68$ & $0.30 \pm 0.47$ & $1.91 \pm 3.46$ & $0.29 \pm 0.48$ & NS \\
\hline weekly npCCr (L/wk/1.73 m²) & $76 \pm 33$ & $48 \pm 13$ & $48 \pm 13$ & $40 \pm 11$ & 0.058 \\
\hline weekly nGFR (L/wk/1.73 m²) & $23 \pm 31$ & $12 \pm 22$ & $39 \pm 59$ & $9 \pm 15$ & NS \\
\hline $\begin{array}{l}\text { no. of patients who had urine } \\
\text { output }>100 \mathrm{~mL} / \text { day }\end{array}$ & 4 & 3 & 3 & 4 & NS \\
\hline $\mathrm{UF}$ (mL/day) & $406 \pm 155$ & $510 \pm 438$ & $405 \pm 225$ & $612 \pm 142$ & NS \\
\hline nPNA (g/kg/day) & $0.91 \pm 0.31$ & $1.17 \pm 0.28$ & $1.38 \pm 0.99$ & $0.80 \pm 0.25$ & NS \\
\hline no. of patients who were & 2 & 1 & 1 & 1 & NS \\
\hline
\end{tabular}

${ }^{a}$ Data are reported as mean $\pm \mathrm{SD}$. Abbreviations used: $\mathrm{npCCr}=$ normalized peritoneal creatinine clearance; nGFR $=$ normalized glomerular filtration rate $\mathrm{nPNA}=$ normalized protein nitrogen appearance; $\mathrm{NS}=$ not significant; $\mathrm{PDE}=$ peritoneal dialysate effluent; $\mathrm{pKt} / V_{\mathrm{urea}}=\mathrm{peritoneal} \mathrm{Kt} / V_{\mathrm{urea}} ; \mathrm{rKt} / V_{\mathrm{urea}}=\mathrm{renal}$ $\mathrm{Kt} / V_{\text {urea }} ; \mathrm{UF}=$ ultrafiltration.

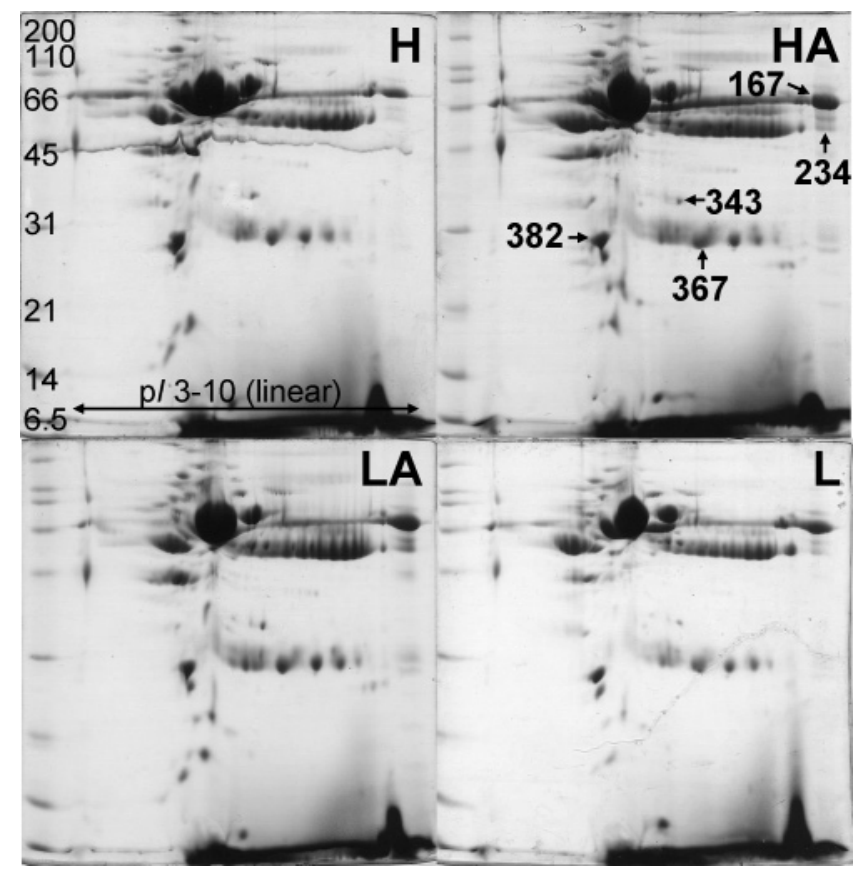

Figure 1. Representative 2-D gel images of the PDE proteins derived from different types of peritoneal membranes. Proteins were precipitated with $75 \%$ ethanol, and an equal amount of total protein $(200 \mu \mathrm{g})$ obtained from each patient was resolved in each 2-D gel ( $n=5$ gels for each group; total $n=20$ ). The resolved protein spots were visualized by CBB-R250 stain. Quantitative intensity analysis and ANOVA with Tukey's posthoc multiple comparisons revealed five protein spots whose intensity levels significantly differed among groups (see Table 2). These protein spots (labeled with numbers) were subsequently identified by MALDI-Q-TOF MS and MS/MS analyses (see also Table 3 and Supporting Information Table S1).

greater nPNA than those who had inadequate dialysis ( $\mathrm{tKt} / V_{\text {urea }}$ $<1.7)(1.17 \pm 0.60$ vs $0.74 \pm 0.19 \mathrm{~g} / \mathrm{kg} /$ day, respectively, $p=$ 0.026).

Figure 1 shows representative 2-D gels of proteins derived from the PDE of individual groups. PDE proteins were concentrated by $75 \%$ ethanol precipitation, which offered a consistent amount of total protein recovered (with a coefficient of variation of $5.58 \%$ from eight independent experiments of the same PDE sample). Quantitative intensity analysis using ANOVA with Tukey's posthoc multiple comparisons of the data obtained from the four different groups (including H, HA, LA and L) revealed five protein spots whose intensity levels significantly differed among groups (Table 2). These proteins were then successfully identified by MALDI-Q-TOF MS and MS/MS analyses (Table 3 and Supporting Information Table S1). The L and LA transporters had lower levels of complement component C4A, immunoglobulin $\kappa$ light chain VLJ region, and chain A, crystal structure of lipid-free human apolipoprotein A-1 (apoA-I), but a higher level of human serum albumin complex with myristic acid and triiodobenzoic acid, when compared to the $\mathrm{H}$ and HA transporters. These data indicate that the transport of macromolecules from the circulation into the PDE is determined by the intrinsic permeability of the peritoneal membranes.

Table 4 shows subgroup analyses of the five proteins whose abundance levels in PDE significantly differed among groups. Parameters that were evaluated included numbers of peritonitis episodes, urea clearance as determined by total (renal and peritoneal) Kt $/ V_{\text {urea, }}$ and nPNA. Patients who had no peritonitis after 1 year follow-up and those who had poorer dialysis adequacy tended to have lower levels of immunoglobulin $\kappa$ light chain VLJ region in their PDE.

The proteomic data were then confirmed by ELISA to measure PDE levels of C4A and IgG in the validation set of the PDE samples obtained from the other 24 patients $(n=6$ in each transport group). Figure 2 clearly show that the differences in PDE abundance of C4A and IgG obtained from ELISA were consistent with those obtained from 2-DE approach.

\section{Discussion}

This is the first preliminary study of the proteome profile of PDE obtained from patients with end-stage renal disease who have different types of peritoneal membranes as determined by the standard PET. The PDE proteome profile is similar but not identical to the urinary proteome profile previously reported in several studies, ${ }^{8-10}$ indicating that CPD can partially work as an artificial kidney, but cannot completely replace the native kidney, a sophisticated organ for maintaining the normal physiology or homeostasis. 
Table 2. Quantitative Data and Statistical Analyses of Protein Spots Whose Intensity Levels Significantly Differed among Groups ${ }^{a}$

\begin{tabular}{|c|c|c|c|c|c|c|c|c|c|c|c|}
\hline \multirow[b]{2}{*}{ spot } & \multicolumn{5}{|c|}{ spot intensity (arbitrary unit) } & \multicolumn{6}{|c|}{ Tukey's posthoc multiple comparisons } \\
\hline & $\mathrm{H}$ & HA & LA & $\mathrm{L}$ & ANOVA & $\mathrm{H}$ vs HA & $\mathrm{H}$ vs $\mathrm{LA}$ & H vs $\mathrm{L}$ & HA vs LA & HA vs L & LA vs L \\
\hline 167 & $1.6335 \pm 0.4964$ & $4.2098 \pm 0.1441$ & $6.0984 \pm 0.7302$ & $5.0756 \pm 0.9573$ & $P=0.001$ & NS & $P=0.001$ & $P=0.009$ & NS & NS & NS \\
\hline 234 & $0.0917 \pm 0.0620$ & $0.9504 \pm 0.1315$ & $1.0418 \pm 0.1537$ & $0.5125 \pm 0.0722$ & $P<0.001$ & $P<0.001$ & $P<0.001$ & NS & NS & NS & $P=0.019$ \\
\hline 343 & $0.2262 \pm 0.0344$ & $0.3092 \pm 0.0341$ & $0.2192 \pm 0.0178$ & $0.1443 \pm 0.0098$ & $P=0.004$ & NS & NS & NS & NS & $P=0.002$ & NS \\
\hline 367 & $2.3479 \pm 0.2399$ & $2.0230 \pm 0.1213$ & $2.0280 \pm 0.2406$ & $1.3296 \pm 0.2905$ & $P=0.042$ & NS & NS & $P=0.031$ & NS & NS & NS \\
\hline 382 & $2.4356 \pm 0.3488$ & $2.0422 \pm 0.0922$ & $1.6401 \pm 0.1240$ & $1.7357 \pm 0.1019$ & $P=0.048$ & NS & $P=0.051$ & NS & NS & NS & NS \\
\hline
\end{tabular}

Table 3. Q-TOF MS and MS/MS Analyses of Proteins Whose PDE Levels Significantly Differed among Groups

\begin{tabular}{|c|c|c|c|c|c|c|c|c|c|c|c|c|}
\hline \multirow[b]{2}{*}{ spot } & \multirow[b]{2}{*}{ protein identity } & \multirow[b]{2}{*}{ NCBI ID } & \multicolumn{3}{|c|}{ MS } & \multicolumn{3}{|c|}{ MS/MS } & \multicolumn{2}{|c|}{$\begin{array}{l}\text { theoretical } \\
\mathrm{pI} / \mathrm{MW}\end{array}$} & \multicolumn{2}{|c|}{$\begin{array}{l}\text { position in } \\
2-\mathrm{D} \text { gel }^{d}\end{array}$} \\
\hline & & & score $^{a}$ & $\% \operatorname{cov}^{b}$ & $\begin{array}{c}\text { no. of } \\
\text { matched } \\
\text { masses }\end{array}$ & $\operatorname{score}^{c}$ & $\% \operatorname{cov}^{b}$ & $\begin{array}{c}\text { no. of } \\
\text { matched } \\
\text { masses }\end{array}$ & pI & MW (kDa) & $\mathrm{pI}$ & $\begin{array}{l}\text { MW } \\
(\mathrm{kDa})\end{array}$ \\
\hline 167 & $\begin{array}{l}\text { human serum albumin in a complex } \\
\text { with myristic acid and triiodobenzoic acid }\end{array}$ & gi|4389275 & 233 & 50 & 27 & 178 & 9 & 4 & 5.69 & 67.99 & 9.50 & 72 \\
\hline 234 & $\alpha 1$-antitrypsin & gi|1942629 & 125 & 46 & 14 & 83 & 3 & 1 & 5.37 & 44.28 & 9.48 & 54 \\
\hline 343 & complement component C4A & gi| 179674 & 92 & 12 & 17 & 61 & 1 & 1 & 6.65 & 194.34 & 7.02 & 36 \\
\hline 367 & immunoglobulin $\kappa$ light chain VLJ region & gi|21669467 & 74 & 43 & 7 & 181 & 19 & 3 & 6.73 & 28.72 & 7.39 & 29 \\
\hline 382 & chain A, crystal structure of lipid-free & gi|90108664 & 149 & 51 & 13 & 227 & 24 & 5 & 5.27 & 28.06 & 5.69 & 30 \\
\hline
\end{tabular}

MS matching score $>65$ was required for significant MS hit. $b \% \operatorname{cov}$ (sequence coverage) $=$ [number of the identified residues/total number of amino acid residues in the protein sequence] $\times 100 \% .{ }^{c}$ MS/MS ion score $>36$ was required for significant MS/MS hit. ${ }^{d}$ Based on the calculation using Image Master $2 \mathrm{D}$ Platinum software.

Table 4. Subgroup Analyses of Proteins Whose PDE Levels Significantly Differed among Groups ${ }^{a}$

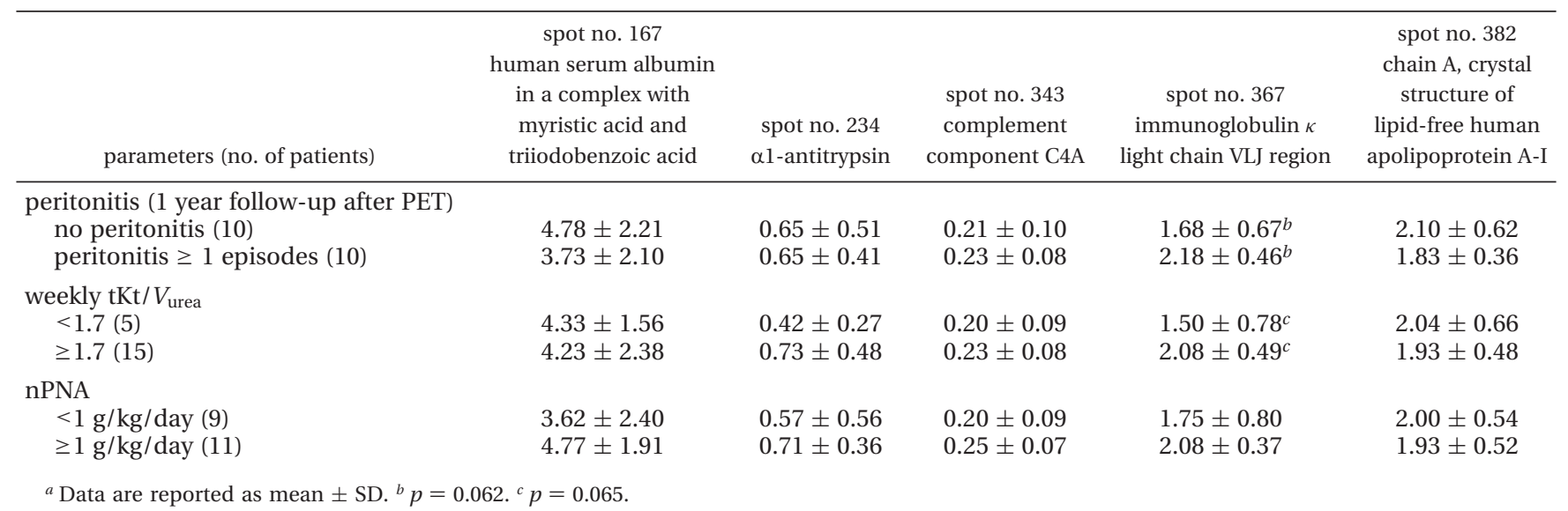

Although the positions of immunoglobulin $\kappa$ light chain VLJ region and apoA-I in the 2-DE gel were consistent with their theoretical pIs and molecular masses, the other three identified proteins had some degrees of inconsistency between the theoretical and actual (in-gel) values (Table 3). This might be simply explained that we identified some of the cleavage, proteolytic, or post-translationally modified products of these proteins, which can alter the proteins' pIs and/or molecular masses. For example, the identified C4A spot (no. 343) was present in the 2-DE gel at approximately $36 \mathrm{kDa}$, whereas its theoretical mass is $194.34 \mathrm{kDa}$ (Figure 1 and Table 3). The MS and MS/MS sequence data clearly showed that we had identified the C-terminal fragment (from residue 1031-1744), not the full length, of C4A (see Table S1). Similarly, chemical adducts could also shift the pI of albumin from its theoretical value, while its molecular mass was slightly changed or almost unaffected (spot no. 167; see Figure 1 and Table 3).

Interestingly, the removal of total protein, mostly albumin, ${ }^{11}$ into the PDE was greater in the high transporters ( $\mathrm{H}$ and $\mathrm{HA}$ ) (Table 1). However, the level of human serum albumin in a complex with myristic acid and triiodobenzoic acid was greater in the low transporters (L and LA) when compared to the higher transporters ( $\mathrm{H}$ and HA). Recently, Ghiggeri and colleagues ${ }^{12,13}$ have shown that repetitive fragmentation products and oxidation forms of albumin are associated with activity of glomerular diseases and degree of nephrotic syndrome. These data strengthen our disparate results, which might imply the potential role of the modified or complexed forms of albumin in peritoneal membrane transport. However, it should be noted that the identification of human serum albumin in a complex 

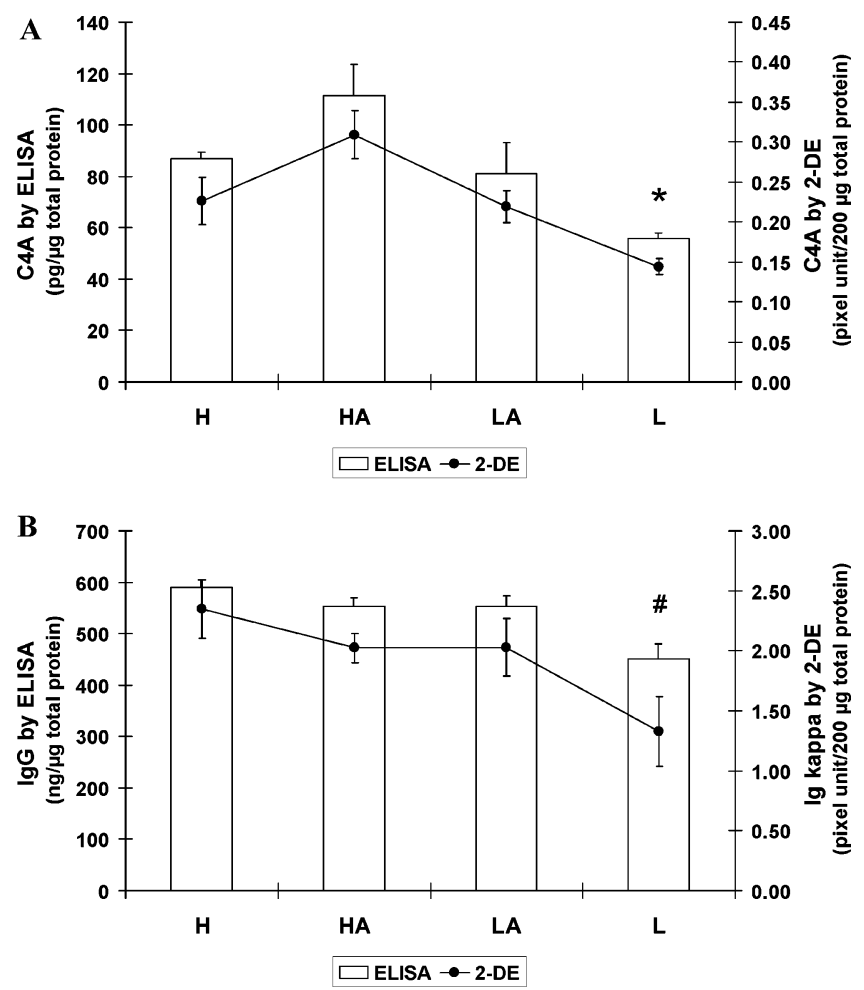

Figure 2. Measurements of PDE levels of C4A (A) and IgG (B) using ELISA in a validation set of 24 patients. The data obtained from ELISA were consistent with the data obtained from 2-DE proteomic analysis. The data are reported as mean \pm SEM $(n=$ 6 for each bar). $*=p<0.005 \mathrm{~L}$ vs HA for both ELISA and 2-DE analyses. \# $=p<0.02 \mathrm{~L}$ vs other groups for ELISA, whereas $p<$ $0.04 \mathrm{~L}$ vs $\mathrm{H}$ for $2-\mathrm{DE}$.

with myristic acid and triiodobenzoic acid was based entirely on peptide mass and ion searches using the MACOT tool. A confirmatory study is required to explore further the role of this complexed form of albumin in peritoneal membrane transport.

The PDE level of C4A was higher in the $\mathrm{H}$ and HA transporters. Although we identified only a C-terminal fragment of C4A to have differential levels by 2-DE (Figure 1, Table 3, and Supporting Information Table S1), the data obtained from ELISA clearly confirmed our proteomic data (Figure 2). C4A is one among several forms of human complement component C4, which plays a crucial role in early step of the classical pathway of complement activation. ${ }^{14}$ Normally, complement component products in peritoneal fluid are most likely activated via the alternative pathway, whereas the PDE C4A, a component of the classical pathway, is usually originated from vascular leakage. ${ }^{15}$ This might explain why the low transporters had lower C4A level in PDE and there was no significant difference of the C4A spot intensity between the peritonitis and peritonitis-free patients.

In contrast, the PDE abundance of immunoglobulin $\kappa$ light chain VLJ region tended to be higher in patients who had peritonitis. It is generally known that immunoglobulin light chains, both $\kappa$ and $\lambda$, can disturb two essential neutrophil functions, including chemotaxis and activation of cellular glucose uptake, and can also attenuate neutrophil apoptosis, the coordinated cell death that is crucial for the resolution of inflammation without tissue destruction. ${ }^{16}$ The higher level of immunoglobulin $\kappa$ light chain VLJ region in PDE of CPD patients with peritonitis might be related to the poorer functions of neutrophils, thereby a higher risk of peritonitis.

$\alpha 1$-Antitrypsin is a small glycoprotein, which is normally produced from the liver and functions as a serine protease inhibitor (serpin), particularly against neutrophil elastase. ${ }^{17}$ Monocytes can also produce $\alpha 1$-antitrypsin and may be the other source of $\alpha 1$-antitrypsin in the PDE. ${ }^{17} \alpha 1$-Antitrypsin can increase by 4 -fold in the plasma of patients with acute-phase states. ${ }^{17}$ This serpin inhibits elastase activity and synthesis of platelet activating factor in the PDE of peritonitis-free patients but had no action in acute infectious peritonitis because of the oxidative inactivation from the proteolytic injury and inflammatory mediators during peritonitis. ${ }^{18} \alpha 1$-Antitrypsin level in the PDE did not differ between patients with peritonitis and those without peritonitis probably because we performed proteomic analysis when all patients were free from peritonitis for more than 1 month, while the normal circulatory half-life of $\alpha 1$-antitrypsin is only 6 days. ${ }^{17}$ Analyzing the PDE proteome during peritonitis may yield differential levels of $\alpha 1$-antitrypsin in patients with and without peritoneal infection.

Patients with $\mathrm{H}$ and HA transport also had higher dialysate apolipoprotein A-I (apoA-I) compared to the L and LA transporters. ApoA-I is the major constituent of high-density lipoprotein (HDL) that plays an important role in reverse cholesterol transport, a process in which apoA-I acts as an acceptor for sequential transfer of phospholipids and free cholesterol from peripheral tissues and transport of cholesterol to the liver and other tissues for excretion and steroidogenesis. ${ }^{19}$ All plasma lipoproteins can appear in the PDE similar to other macromolecules. ${ }^{20}$ The peritoneal loss of HDL (may be up to $34 \%$ of its synthetic rate) is inversely correlated with the plasma HDL level. ${ }^{20} \mathrm{~A}$ considerable loss of apoA-I in the PDE had been previously reported and had a tendency to decrease the plasma levels of apoA-I and HDL although they were not statistically significant. ${ }^{21}$ This may be the reason that why the high solute transporters are prone to develop atherosclerosis. ${ }^{22}$

In summary, five proteins were removed with differential proportions into the PDE of CPD patients who had different types of peritoneal membranes. The high transporters tended to have greater amount of total protein, which indicated the protein loss into the PDE. The higher level of $\kappa$ light chain may be an indicator or may play an important role in peritonitis in CPD patients. However, the number of subjects in our present study was relatively small. Extending the study, prospectively and longitudinally, with a larger number of patients and with subgroup analyses may yield additional information of the dialysate proteins in association with dialysis adequacy and its complications.

Abbreviations: 2-DE, two-dimensional gel electrophoresis; ACN, acetonitrile; apoA-I, apolipoprotein A-I; BSA, bovine serum albumin; CHAPS, 3-[(3-cholamidopropyl)dimethylamino]1-propanesulfonate; CHCA, $\alpha$-cyano-4-hydroxycinnamic acid; $\mathrm{CPD}$, chronic peritoneal dialysis; Cr, creatinine; DTT, dithiothreitol; $\mathrm{H}$, high transport rate; $\mathrm{HA}$, high average transport rate; L, low transport rate; LA, low average transport rate; MALDIQ-TOF, matrix-assisted laser desorption ionization quadrupole time-of-flight; nGFR, normalized glomerular filtration rate; npCCr, normalized peritoneal creatinine clearance; nPNA, normalized protein nitrogen appearance; PDE, peritoneal dialysate effluents; PET, peritoneal equilibration test; $\mathrm{pKt} / V_{\text {urea, }}$, peritoneal urea clearance normalized with distribution volume of urea; $\mathrm{rKt} / V_{\text {urea, }}$ renal urea clearance normalized with distribution volume of urea; SDS, sodium dodecyl sulfate; TFA, 
trifluoroacetic acid; $\mathrm{tKt} / V_{\text {urea }}$, total urea clearance normalized with distribution volume of urea; UF, ultrafiltration.

Acknowledgment. This study was supported by Mahidol University, the National Research Council of Thailand, the National Center for Genetic Engineering and Biotechnology, a Siriraj Grant for Research and Development, the Commission on Higher Education, and The Thailand Research Fund (to V. Thongboonkerd). T. Semangoen is supported by a Siriraj Graduate Scholarship.

Supporting Information Available: Peptide mass fingerprint and sequencing data of the identified proteins using MALDI-Q-TOF MS and MS/MS analyses (Table S1). This material is available free of charge via the Internet at http:// pubs.acs.org.

\section{References}

(1) Twardowski, Z. J. Clinical value of standardized equilibration tests in CAPD patients. Blood Purif. 1989, 7, 95-108.

(2) Brimble, K. S.; Walker, M.; Margetts, P. J.; Kundhal, K. K.; Rabbat, C. G. Meta-analysis: peritoneal membrane transport, mortality, and technique failure in peritoneal dialysis. J. Am. Soc. Nephrol. 2006, 17, 2591-2598.

(3) Davies, S. J. Mitigating peritoneal membrane characteristics in modern peritoneal dialysis therapy. Kidney Int. Suppl. 2006, S76S83.

(4) Chung, S. H.; Heimburger, O.; Stenvinkel, P.; Wang, T.; Lindholm, $B$. Influence of peritoneal transport rate, inflammation, and fluid removal on nutritional status and clinical outcome in prevalent peritoneal dialysis patients. Peritoneal Dial. Int. 2003, 23, 174183.

(5) Dombros, N.; Dratwa, M.; Feriani, M.; Gokal, R.; Heimburger, O.; Krediet, R.; Plum, J.; Rodrigues, A.; Selgas, R.; Struijk, D.; Verger, C. European best practice guidelines for peritoneal dialysis. 7 . Adequacy of peritoneal dialysis. Nephrol. Dial. Transplant. 2005, 20 (Suppl. 9), ix24-ix27.

(6) Dombros, N.; Dratwa, M.; Feriani, M.; Gokal, R.; Heimburger, O.; Krediet, R.; Plum, J.; Rodrigues, A.; Selgas, R.; Struijk, D.; Verger, C. European best practice guidelines for peritoneal dialysis. 8 . Nutrition in peritoneal dialysis. Nephrol. Dial. Transplant. 2005, 20 (Suppl. 9), ix28-ix33.

(7) O'Malley, R. Life's (more than) a BLAST. Biochemist 2002, 24, $21-23$.

(8) Thongboonkerd, V.; McLeish, K. R.; Arthur, J. M.; Klein, J. B. Proteomic analysis of normal human urinary proteins isolated by acetone precipitation or ultracentrifugation. Kidney Int. 2002, 62, 1461-1469.
(9) Oh, J.; Pyo, J. H.; Jo, E. H.; Hwang, S. I.; Kang, S. C.; Jung, J. H.; Park, E. K.; Kim, S. Y.; Choi, J. Y.; Lim, J. Establishment of a nearstandard two-dimensional human urine proteomic map. Proteomics 2004, 4, 3485-3497.

(10) Pieper, R.; Gatlin, C. L.; McGrath, A. M.; Makusky, A. J.; Mondal, M.; Seonarain, M.; Field, E.; Schatz, C. R.; Estock, M. A.; Ahmed, N.; Anderson, N. G.; Steiner, S. Characterization of the human urinary proteome: a method for high-resolution display of urinary proteins on two-dimensional electrophoresis gels with a yield of nearly 1400 distinct protein spots. Proteomics 2004, 4, 1159-1174.

(11) Kabanda, A.; Goffin, E.; Bernard, A.; Lauwerys, R.; van Ypersele, d. S. Factors influencing serum levels and peritoneal clearances of low molecular weight proteins in continuous ambulatory peritoneal dialysis. Kidney Int. 1995, 48, 1946-1952.

(12) Candiano, G.; Musante, L.; Bruschi, M.; Petretto, A.; Santucci, L.; Del Boccio, P.; Pavone, B.; Perfumo, F.; Urbani, A.; Scolari, F.; Ghiggeri, G. M. Repetitive fragmentation products of albumin and alpha1-antitrypsin in glomerular diseases associated with nephrotic syndrome. J. Am. Soc. Nephrol. 2006, 17, 3139-3148.

(13) Musante, L.; Candiano, G.; Petretto, A.; Bruschi, M.; Dimasi, N.; Caridi, G.; Pavone, B.; Del Boccio, P.; Galliano, M.; Urbani, A.; Scolari, F.; Vincenti, F.; Ghiggeri, G. M. Active focal segmental glomerulosclerosis is associated with massive oxidation of plasma albumin. J. Am. Soc. Nephrol. 2007, 18, 799-810.

(14) O'Neill, G. J.; Yang, S. Y.; Dupont, B. Two HLA-linked loci controlling the fourth component of human complement. Proc. Natl. Acad. Sci. U.S.A. 1978, 75, 5165-5169.

(15) Young, G. A.; Kendall, S.; Brownjohn, A. M. Complement activation during CAPD. Nephrol. Dial. Transplant. 1993, 8, 1372-1375.

(16) Cohen, G. Immunoglobulin light chains in uremia. Kidney Int. Suppl. 2003, S15-S18.

(17) Carrell, R. W. alpha 1-Antitrypsin: molecular pathology, leukocytes, and tissue damage. J. Clin. Invest. 1986, 78, 1427-1431.

(18) Mariano, F.; Tetta, C.; Montrucchio, G.; Cavalli, P. L.; Camussi, G. Role of alpha 1-proteinase inhibitor in restraining peritoneal inflammation in CAPD patients. Kidney Int. 1992, 42, 735-742.

(19) Ajees, A. A.; Anantharamaiah, G. M.; Mishra, V. K.; Hussain, M. M.; Murthy, H. M. Crystal structure of human apolipoprotein A-I: insights into its protective effect against cardiovascular diseases. Proc. Natl. Acad. Sci. U.S.A. 2006, 103, 2126-2131.

(20) Kagan, A.; Bar-Khayim, Y.; Schafer, Z.; Fainaru, M. Kinetics of peritoneal protein loss during CAPD: II. Lipoprotein leakage and its impact on plasma lipid levels. Kidney Int. 1990, 37, 980-990.

(21) Querfeld, U.; LeBouef, R.; Salusky, I.; Fine, R. Lipoproteins in prediatric patients treated with CAPD/CCPD. Kidney Int. 1988, 33, 248.

(22) Sezer, S.; Tutal, E.; Arat, Z.; Akcay, A.; Celik, H.; Ozdemir, F. N.; Haberal, M. Peritoneal transport status influence on atherosclerosis/inflammation in CAPD patients. J. Renal Nutr. 2005, 15, 427-434.

PR0702969 